\title{
El asesoramiento vocacional y profesional a través del Self-Directed Search (SDS)
}

\author{
José Manuel Martínez Vicente
}
Departamento de Psicología Evolutiva y de la Educación Universidad de Almería

\section{España}

jvicente@ual.es 


\section{Resumen}

El Self-Directed Search es una guía para la planificación educativa y profesional que a diferencia de otros inventarios de intereses vocacionales es autoaplicable, autopuntuable y autointerpretable. El objetivo de este artículo es realizar un análisis y descripción de las principales aplicaciones que tiene el Self-Directed Search en el ámbito del desarrollo de la carrera y del asesoramiento vocacional y profesional. Para ello se realiza una revisión de los últimos estudios en los que se ha empleado, se describe cuál fue su origen y desarrollo y se analizan y discuten sus características y sus principales aplicaciones en el ámbito del asesoramiento vocacional y profesional.

Palabras Clave: Self Directed-Search, tipología de Holland, elección vocacional, SDS 


\section{Introducción}

El Self-Directed Search (SDS) puede considerarse una guía para la planificación de la carrera que funciona de forma similar a un inventario de intereses vocacionales y profesionales. A diferencia de otros instrumentos que evalúan los intereses, el SDS es autoadministrable, autopuntuable y autointerpretable por los sujetos que lo cumplimentan. Los datos aportados por sus actuales editores, Psychological Assesment Resources (PAR) de Florida (EEUU), señalan que ha sido utilizado por más de 22 millones de personas de todo el mundo en sus más de 30 años de existencia y ha sido traducido a 25 idiomas. En consecuencia, llegan a afirmar que el SDS es uno de los instrumentos de orientación de la carrera más empleados en todo el mundo.

El empleo del Self-Directed Search en el asesoramiento profesional y vocacional ha provocado el que se realicen numerosas investigaciones, desde su aparición, en las que éste se ha utilizado como un instrumento para recabar información relacionada con: los efectos que produce en los usuarios, su utilidad, su relación con variables personales (sexo, edad, raza, inteligencia, personalidad, estilos de pensamiento, nivel académico y cultural), su adaptación a otros idiomas, la validación de la Teoría Tipológica de Holland, su relación con otros inventarios de interés y de personalidad y sobre la propia validez y fiabilidad de su estructura. En definitiva, muchas de las investigaciones han sido diseñadas para incrementar la difusión y la utilidad del SDS (Holland, Fritzsche y Powel, 1994).

Centrándonos en el periodo comprendido entre los años 2000 y 2006 encontramos que el SDS ha sido utilizado en unas 61 investigaciones. Parte de estos estudios se han centrado en la identificación de la estructura de los intereses vocacionales y la validación de la teoría de Holland en otras poblaciones como Croacia (Sverko y Babarovik, 2006), China (Leung y Hou, 2001; Leung y Hou, 2005; Yang, Stokes y Hui, 2005) e Islandia (Einarsdottir, Rounds, Aegisdottir y Gerstein, 2002). Además se han realizado adaptaciones del SDS al alemán (Muck, 2005) y al español (Martínez-Vicente, 2001; Martínez-Vicente y Valls, 2006). También se han desarrollado investigaciones sobre la relación de la congruencia (grado de relación entre los tipos de personalidad medidos a través del SDS y el tipo de ambiente profesional) con otras variables como: la satisfacción profesional en enfermeros/as (Dockins, 2004) y las metas fundamentales en mujeres estudiantes africo-americanas y europeo-americanas (Bath, 2004). 
Otra línea de estudios se ha encargado de obtener perfiles de intereses vocacionales empleando muestras de mujeres carpinteras (Swan, 2005), asistentes médicos (Labarbera, 2005) y estudiantes de ingeniería (Price, 2003). Aparecen otro conjunto de estudios en el que el SDS se emplea con muestras de estudiantes con características especiales, como ocurre en el de Osborn y Reardon (2006) realizado con estudiantes negros de secundaria catalogados como de alto riesgo; el de Glaser et al. (2003) con hombres adolescentes juzgados; y el de Wilkes (2002) con adultos discapacitados.

Por otra parte, se dan otras investigaciones relacionadas con la personalidad y aspectos como: la motivación (Yahney, 2005), los estilos de pensamiento (Balkis e Isiker, 2005; Zhang, 2000; Zhang, 2001), las opciones de carrera no tradicional en mujeres (Hoffman, 2005), la orientación teórica preferida en el asesoramiento de estudiantes (Lowman, Palmer, Santana y Abot, 2003), la aproximación de aprendizaje (Zhang, 2004), la indecisión profesional (Primi et al., 2001) y los desórdenes de la personalidad ( Mahalik, 2001).

A través de otros estudios se ha pretendido relacionar o comparar los resultados obtenidos en el SDS con otras diferentes medidas de interés. Así Jones, Sheffield y Joyner (2000) compararon los efectos del Career Kley, el SDS y el Job-O Enchanced. Por su parte, Savickas, Taber y Spokane (2002) comprobaron la correspondencia de las puntuaciones obtenidas en 5 inventarios de interés: el Campbell Interest and Skills Survey, el Kuder Occupational Interest Survey-Form DD, el Self-Directed Search, el Strong Interest Inventory-Skills Confidence Edition, y el Revised Unisex Edition of the American College Testing Interest Inventory. Autores como Lowman et al. (2003) se interesaron por verificar la posible intercambialidad de los resultados obtenidos empleando el Strong Vocational Interest Blank, el Vocational Preferente Inventory (PCI) y el SDS. Por último, Miller, Springer y Cowger (2004) examinaron la congruencia entre el Counseling Orientation Scale (COS) y el SDS

El empleo de las nuevas tecnologías hace que se planteen estudios como el de Lumsden et al. (2004) en el que se comparan tres formas distintas de administración del SDS: en papel, a través del ordenador e internet; o el de Barak y Cohen (2002) en el que se examina la utilidad de una versión on line del SDS. 
El SDS fue desarrollado por Holland en 1971 y desde esa fecha ha sido revisado tres veces, siendo la revisión de 1994 la más reciente. Es preciso destacar que este instrumento es fruto de la Teoría de Elección Vocacional y Profesional desarrollada por Holland (1992, 1997). Por lo tanto, la utilidad del Self-Directed Search (SDS) depende directamente de la utilidad de la citada teoría cuyas principales características comentaremos en un posterior apartado.

En cualquier caso, el objetivo de este artículo es realizar un análisis y descripción de las principales aplicaciones que tiene el Self-Directed Search en el ámbito del desarrollo de la carrera y del asesoramiento vocacional y profesional.

\section{Teoría tipológica de Holland}

Holland $(1975,1981,1992,1997)$ presenta un modelo explicativo parsimonioso, a la vez que sencillo, con el que pretende explicar como las personas toman decisiones vocacionales y profesionales; qué les hace cambiar de empleo o de interés en la elección; y cuáles son los factores, tanto personales como ambientales, que pueden facilitar el logro y satisfacción vocacional y profesional.

Como señalan Martínez-Vicente, Valls y Álvarez (2003) el origen de la teoría se situa en la convicción por parte de Holland de que las personas que tienen diferentes intereses tienen diferentes tipos de personalidad y que la elección de una determinada profesión refleja no sólo la motivación personal, el conocimiento que se tenga de ésta y las habilidades que se posean para su desempeño, sino también refleja características de personalidad. Además asume que muchas profesiones y conductas sociales provienen de la interacción de las personas y el entorno, es decir, personalidad y ambiente laboral interaccionan entre si influyendo el uno en el otro de manera bidireccional. De tal modo que Holland (1997, p.11) llega a decir que "el trabajo cambia a las personas, y las personas cambian los trabajos".

Su teoría parte de las siguientes asunciones:

- La mayoría de las personas se pueden categorizar en uno de los siguientes tipos de personalidad: Realista (R), Investigador (I), Artista (A), Social (S), Emprendedor (E) y Convencional (C). Cada uno de estos tipos representa una síntesis de lo que se sabe de las personas en relación con: actividades preferidas, competencias re- 
queridas, objetivos y valores de vida, autocreencias, estilo de resolución de problemas y características personales. Todos estos aspectos se integran en un modelo teórico con el que se puede comparar cualquier persona para verificar su parecido con cada uno de los tipos.

- Existen seis modelos de ambientes: Realista (R), Investigador (I), Artista (A), Social (S), Emprendedor (E) y Convencional (C). Se parte de la premisa de que los tipos de personalidad que son dominantes en un determinado ambiente son los que proporcionan los aspectos característicos del mismo; de esta manera un ambiente Realista estará dominado por tipos Realistas, en ambiente Convencional estará dominado por tipos Convencionales, etc.

- Las personas buscan ambientes donde pueden ejercer sus destrezas y habilidades, expresar sus actitudes y valores y elegir problemas y roles que sean de su agrado. Esto lleva a que las personas de un determinado tipo de personalidad busquen el ambiente que más se ajuste a ellas. Así un tipo Emprendedor buscará un ambiente Emprendedor para desarrollar su trabajo.

- La conducta de una persona está determinada por la interacción entre su personalidad y las características del ambiente. En este sentido conocidos el patrón de personalidad de una persona y de su ambiente, se pueden pronosticar resultados en relación con el tipo de elección vocacional más probable y el nivel de satisfacción, estabilidad y logro dentro de él.

Una descripción detallada de los tipos de personalidad y modelos ambientales se puede ver en Holland (1997). De manera resumida podemos señalar que: el tipo Realista prefiere tareas relacionadas con la manipulación de objetos, herramientas, máquinas y animales; el tipo Investigador prefiere actividades que conducen a la observación, a lo simbólico, a lo sistemático y a la investigación creativa de los fenómenos físicos, biológicos y culturales para comprenderlos, explicarlos y controlarlos; el tipo Artístico prefiere actividades que implican una manipulación de materiales físicos, verbales y humanos para crear formas y productos; el tipo Social prefiere actividades que implican la interacción con otras personas con al finalidad de informar, curar, educar e instruir; el tipo Emprendedor prefiere actividades que suponen la manipulación de otros para conseguir objetivos organizativos y ganancias económicas; el tipo Convencional prefiere actividades que implican una sistemática, ordenada y explícita manipulación de datos en registros y archivos, reproducir materiales, organizar datos numéricos conforme a un plan determinado, trabajar con máquinas de oficina y de procesamiento de 
textos para la consecución de objetivos de tipo económico y organizativo. Por otro lado, las demandas y oportunidades que caracterizan a los modelos ambientales están relacionadas con las tareas preferidas por los respectivos tipos de personalidad.

Para evaluar los tipos de personalidad Holland elabora el Vocational Preferente Inventory (Holland, 1958) y el Self-Directed Search (Holland, 1971). Ambos instrumentos cuentan un procedimiento sencillo para clasificar las personas empleando seis escalas; proporcionando un código formado por la combinación de los seis tipos en el que se tiene en cuenta el orden en las puntuaciones obtenidas en cada uno de ellos. En relación con los modelos ambientales Gottfredson y Holland (1991) desarrollan el Position Classification Inventory que representa un método para clasificar los ambientes ocupacionales de acuerdo con la clasificación teórica de los tipos.

Otro de los aspectos a destacar de esta teoría está relacionado con el descubrimiento de la estructura Hexagonal de relación entre los tipos. Es decir, los tipos de interés configuran un hexágono en cuyos vértices se sitúan los tipos de acuerdo con el siguiente orden: RIASEC, de modo que los tipos más próximos están más relacionados entre sí y asumiendo que las relaciones entre los tipos son inversamente proporcionales a las distancias que los separan.

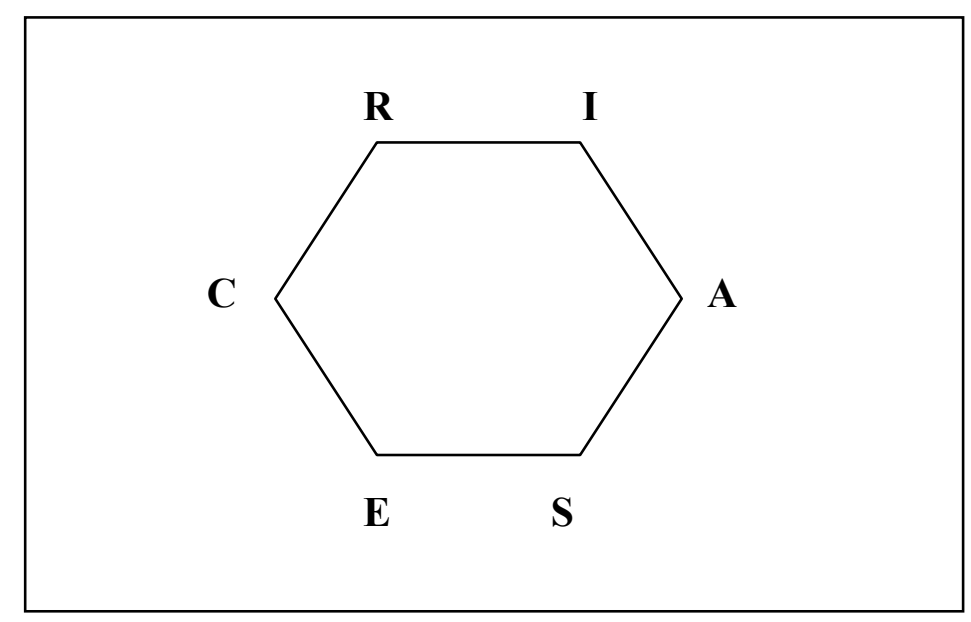

Figura 1. Modelo hexagonal de relación entre los tipos de personalidad y modelos ambientales

Este modelo hexagonal representa un instrumento de cálculo para la teoría, permitiendo definir y verificar unos supuestos secundarios como son la consistencia, la congruencia y la diferenciación; que van a actuar como indicadores empíricos de la probabilidad de realizar elecciones profesionales adecuadas y estables y, en consecuencia, incrementar la posibilidad 
de obtener una mayor satisfacción y logro en el trabajo. Para Spokane (1996) este modelo viene a confirmar las afirmaciones teóricas de la disposición de los intereses así como permitir una mejor comprensión del sistema organizativo de Holland.

\section{EI Self-Directed Search (SDS)}

\section{Materiales}

El Self-Directed Search (SDS) en su Forma R se compone de tres cuadernos (Holland, 1995a, 1995b, 1995c): el Cuaderno de Evaluación (The Assesement booklet), el Descubridor de ocupaciones (The Occupations finder) y Usted y su carrera (You and your career) diseñados para ser usados con estudiantes de Secundaria (High Schol), estudiantes universitarios y adultos. Hay otras formas del SDS, cada una específica para una población determinada. Estas versiones comprenden la versión Fácil (Form E), la versión Planificación de la Carrera (Form PC) y, por último, la versión de Exploración de la Carrera (Career Explorer).

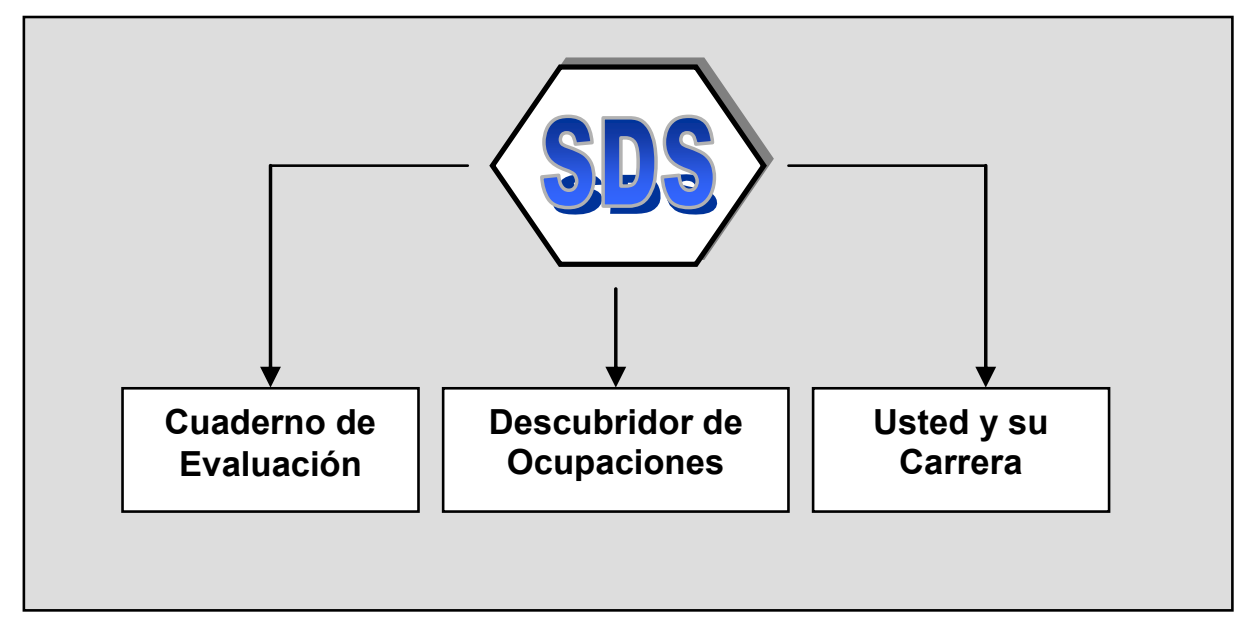

Figura 2. Materiales que componen el SDS Form R

1) Cuaderno de Evaluación (The Assessement booklet).

Es autoadministrable y autopuntuable. Se usa para evaluar las puntuaciones en cada una de las secciones que componen el SDS (Actividades, Habilidades, Ocupaciones y Autoevaluaciones) y deriva en un Código Sumario o Resumen que sintetiza el parecido de una persona con los seis tipos de personalidad establecidos por Holland. La persona usa este 
Código Sumario para buscar en el Descubridor de Ocupaciones (clasifica 1335 ocupaciones) aquellas ocupaciones que se ajusten a su código de personalidad.

En el Cuaderno de Evaluación podemos apreciar tres partes bien diferenciadas. En la primera, se pide al sujeto que señale cuáles son sus aspiraciones vocacionales. Su origen viene determinado por un estudio de Holland (1963) en el que constató que las aspiraciones vocacionales, especialmente las más recientes, eran buenas estimaciones de lo que las personas podrían escoger o hacer en un futuro cercano $\mathrm{y}$, por consiguiente, consideró que éstas podían tener un buen nivel predictivo al respecto. La inclusión en esta sección para Holland, Fritzsche y Powell (1994) tiene dos objetivos:

a) Predecir la ocupación futura de una persona, ya que la categoría de la elección anunciada por una persona es tan eficaz como cualquier otro dispositivo psicológico.

b) Proporcionar una comprobación de la validez del código sumario.

La segunda, escalas SDS y puntuaciones, se utiliza para obtener una puntuación en cada escala, respondiendo a una serie de ítems relacionados con: actividades que les gustaría hacer; competencias relacionadas con actividades que creen pueden o podrían hacer de forma competente; ocupaciones que les interesa o le llaman la atención. Todo ello referido a cada uno de los tipos de personalidad definidos. Por otra parte, se cuenta con una sección de autoevaluaciones en la que el sujeto debe evaluar el grado de habilidad o destreza que posee comparándose con las personas de su misma edad.

La tercera parte se emplea para explicar el significado del código sumario obtenido ofreciéndose, además, unas orientaciones y libros que pueden ser de utilidad.

Las partes anteriormente citadas se materializan en el Cuaderno de Evaluación, generando las siguientes secciones: Aspiraciones Ocupacionales, Escalas SDS y puntuaciones (Actividades, Competencias, Ocupaciones y Autoevaluaciones), Cómo organizar sus respuestas, Qué significa su código sumario, Algunos pasos a tomar y algunos libros útiles.

2) El Descubridor de ocupaciones (The Occupations Finder). 
El Cuaderno de Clasificación es producto de la clasificación ocupacional propuesta a priori por la teoría de Holland (1959). En su origen, fue definido empíricamente usando los perfiles VPI (Vocational Preferences Inventory) de aspirantes a diferentes ocupaciones; posteriormente se integraron datos ocupacionales obtenidos del Strong (Campbell y Holland, 1972) y los datos Pardue de 879 ocupaciones (McCormick, Jeanneret y Mechan, 1972).

Incluye las 1.335 profesiones u ocupaciones más comunes de los Estados Unidos. Las profesiones u ocupaciones están agrupadas de acuerdo a tipos y subtipos (vg. dentro del tipo $\mathbf{S}$ nos podemos encontrar el subtipo SA y dentro de éste el SAI). Cada subtipo de ocupación está ordenado en consonancia con el nivel de educación requerido por la misma de acuerdo al Diccionario de Títulos Ocupacionales (DOT; Gottfredson y Holland, 1989).

3) Cuaderno interpretativo: Usted y su carrera.

Fue desarrollado para que el Self-Directed Search (SDS) proporcionase una experiencia más completa y más autodirigida. Tiene como pretensión el liberar al personal orientador de la rutina y el gasto de tiempo explicando la tipología y ocupándose de las interpretaciones y problemas más comunes que pueden ser resueltos mediante su lectura.

En él se ofrece, de manera sencilla, un resumen de las ideas científicas que apoyan el uso de estos inventarios, se enseña a usar e interpretar los códigos y puntuaciones obtenidos en el Cuaderno de Evaluación y se ofrecen algunas ideas para tomar buenas decisiones sobre la carrera. Los informes de los consejeros u orientadores sugieren que este cuaderno informativo, cuando es leído por el que realiza el Cuaderno de Evaluación, reduce las preguntas y aumenta la satisfacción del que lo usa.

\section{Origen y desarrollo del Self-Directed Search (SDS).}

El origen del SDS hay que situarlo en el propio desarrollo de la Teoría Tipológica de Holland y en el de uno de sus primeros instrumentos de evaluación de intereses profesionales: el Vocational Preference Inventory (VPI). En la figura 3 podemos observar las distintas fases transcurridas hasta que se formuló el Self-Directed Search. 
Con el Vocational Preference Inventory (VPI) se comprobó que la disposición de los items en escalas cortas, de igual longitud y dispuestas en orden cíclico, hacía al instrumento más fácil de puntuar. Fue necesario el transcurso de 5 años, desde 1953 a 1958, para llegar de escalas de desigual longitud e items dispuestos aleatoriamente a esta simple, pero efectiva, solución.

La clasificación de los tipos propuesta por Holland se inició asumiendo que todas las ocupaciones podrían organizarse en seis grupos paralelos a los seis tipos de personalidad propuestos (R, I, A, S, E, C) por éste (Holland, 1959). Esta idea surge de los resultados obtenidos del análisis de matrices de correlaciones y análisis factoriales de inventarios de intereses y personalidad. Por otra parte, las primeras clasificaciones de los ambientes utilizan las primeras seis escalas del VPI para categorizar las ocupaciones. Más adelante los principales perfiles VPI para ocupaciones y campos de entrenamiento se usaron para crear el código ocupacional de tres letras (Holland, 1966a). Esto lleva a la elaboración del Descubridor de Ocupaciones (The Occupations Finder) que es una versión abreviada del Diccionario Holland de Códigos Ocupacionales (DOT; Gottfredson y Holland, 1989) en donde aparecen catalogadas 1335 ocupaciones según el código de tres letras de Holland.

Otro de los aspectos a destacar es el descubrimiento del modelo hexagonal. La disposición de la clasificación de acuerdo a un modelo hexagonal surge por la necesidad de contar con categorías ocupacionales en un orden que fuera consistente con los datos ocupacionales. Strong (1943) llegó a anticipar el modelo hexagonal, pero fueron Whitney y Holland los que descubren el modelo en una muestra de universitarios de ambos sexos (Holland, Whitney, Cole y Richards, 1969).

Holland aprovechó el modelo hexagonal para definir e integrar los principales conceptos (congruencia y consistencia) de su teoría, que en esos momentos estaba en revisión. En lo que respecta a las ocupaciones se organizaron primeramente en seis categorías principales (RIASEC); después se asumió que las categorías se podrían organizar en subcategorías siguiendo el orden del modelo hexagonal RI, RA, RS, RE, RC,... y a su vez en otras RIA, RIS, RIE, RIC,... 


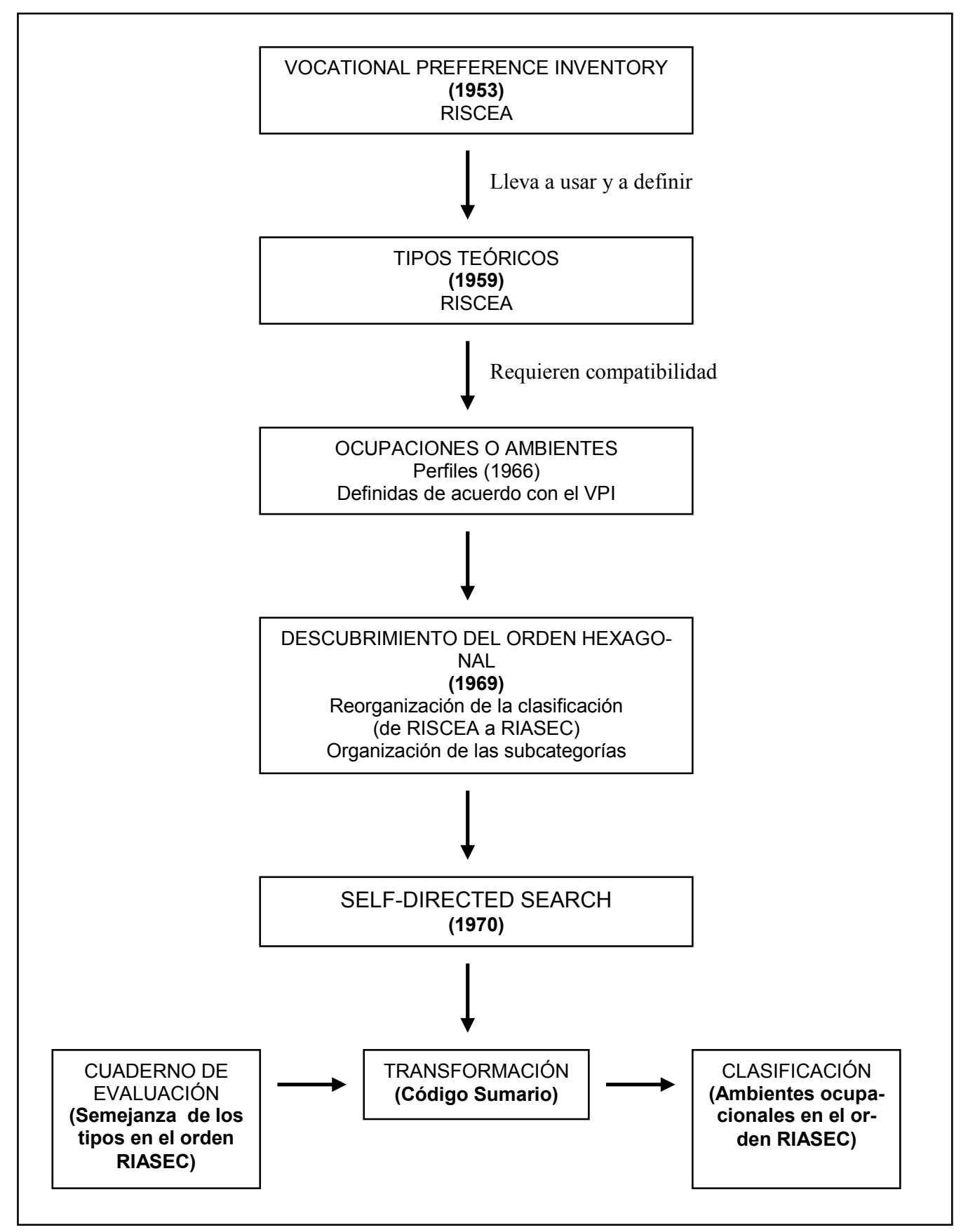

Figura 4. Origen y desarrollo del Self-Directed Search (Holland et al, 1994)

El SDS tuvo su origen en una demanda que Thomas Magoon hace en 1970 a Holland, en la que le solicitaba un recurso de orientación profesional para utilizar con universitarios de primer año. En ese tiempo Holland se propuso elaborar una evaluación en la cual las escalas de ámbitos diferentes deberían seguir la teoría explícitamente: seis escalas en cada dominio 
(actividades, competencias, autoestimaciones, ocupaciones), dispuestas en orden RIASEC y de igual longitud. Esta tarea fue acompañada de múltiples análisis de items y estudios teóricos durante el periodo de 1959 a 1969. Desde 1970 a 1977, el proceso de autopuntuación sufrió numerosas revisiones, siendo en este último año cuando se decide que las puntuaciones deberían ser sumadas y expuestas conjuntamente en una única página con la finalidad de disminuir los errores. Hecho que efectivamente se corroboró.

Las aspiraciones vocacionales, otra de las secciones del SDS, tuvieron un origen accidental. Examinando la teoría, Holland descubrió que las aspiraciones vocacionales tenían más valor predictivo que el código más alto obtenido en el Inventario de Preferencias Vocacionales (VPI) o las escalas del Strong Vocational Interest Blank (SVIB). De ahí que tomara la decisión de incluirlo en el SDS. De hecho se introduce para comprobar la validez del código sumario. El código de las aspiraciones vocacionales cuando se usa en combinación con el código del SDS hace que las predicciones sean más efectivas.

Ante las continuas referencias de que el SDS no era tan autointerpretativo como Holland pretendía, éste se propuso entre 1975 y 1976 crear un cuaderno que permitiera al usuario comprender lo esencial de la teoría y no ser tan sólo una descripción de los tipos. Para ello desarrolló dos formas, una corta y otra larga. Tras la comprobación de la utilidad de cada una en diversas muestras optó por la forma corta. Actualmente el cuaderno interpretativo del SDS se denomina "You and you career" (usted y su carrera).

Se han realizado diversas ediciones del Self-Directed Search desde su aparición en 1971 con la finalidad, por un lado, de perfeccionarlo y obtener mayores índices de fiabilidad y validez y, por otro, para adaptarlo a los cambios sufridos en el tiempo. A pesar de que la edición de 1971 contaba con una fiabilidad y validez aceptables se crea la edición de 1977, que aunque era bastante parecida a la edición de 1971 modificó varios items Realistas, para reducir diferencia en cuanto al sexo; se revisaron 2 items de la sección de las competencias y 16 items de la sección de ocupaciones fueron reemplazados por otros nuevos. La siguiente revisión se produce en 1985. Finalmente, la edición de 1994 se parece mucho a la de 1985. Tan sólo 67 de los 228 items fueron revisados, muchos de éstos se reemplazaron por su pérdida de actualidad. 


\section{Características}

El SDS es un instrumento basado en la Teoría Tipológica de elección profesional y vocacional de Holland y que surge fruto de la dimensión práctica de la misma siendo sus principales características el ser un inventario de intereses vocacionales autoaplicable, autopuntuable y autointerpretable. Aspectos que lo distinguen de otras herramientas de orientación vocacional y profesional. Aunque como señalan Holland, Powel y Fritzsche (1994) fue desarrollado para tratar problemas vocacionales se le han encontrado otras aplicaciones relacionadas con la asistencia vocacional, la organización de datos ocupacionales y personales y en la investigación.

Holland y Rayman (1986) citan como características fundamentales del Self-Directed Search (SDS) las siguientes:

1. El incremento de la autocomprensión del número de alternativas vocacionales a tener en cuenta.

2. La simplicidad en la puntuación del inventario.

3. Posibilita la autoexploración comprensiva de las posibilidades ocupacionales y de las características personales.

4. Permite la aplicación, corrección e interpretación sin la presencia de un asesor.

5. Compatibilidad con muchos materiales de orientación vocacional.

6. Es tanto un inventario de intereses como de personalidad.

7. Organiza, dentro de una teoría, y relaciona las aspiraciones vocacionales, los intereses, las competencias y las autoestimaciones de una persona con una clasificación ocupacional.

El SDS se desarrolla para conseguir dos objetivos principales: 1) aumentar el número de personas a las que el orientador puede atender y 2) facilitar una experiencia de orientación vocacional y profesional para personas que no tienen o no desean tener acceso a un orientador o asesor vocacional.

Un aspecto que es destacado por Holland y Rayman (1986) del SDS es que el tiempo de cumplimentación, puntuación e interpretación se reduce, eliminando a veces una innecesaria orientación individualizada; con lo cual, los orientadores disponen de mayor tiempo para 
dedicar a las personas que necesiten un asesoramiento individualizado y para planificar y evaluar programas.

El SDS ha sido utilizado en muchos contextos, como enseñanza secundaria, universidades, centros de adultos, instituciones correccionales, centros de mujeres y oficinas de empleo, para la educación de la carrera y como guía vocacional y de colocación. En los negocios y en la industria ha sido empleado para la colocación, tanto para clasificar el empleo como para comprobar la adecuación al puesto de trabajo a fin de facilitar el desarrollo del personal. El SDS, y también su tipología subyacente, han demostrado ser instrumentos útiles en la investigación llevada a cabo en educación, negocios y ciencias sociales (Holland, Powel y Fritzsche, 1994).

Las investigaciones que se han realizado sobre su uso y la influencia que ejerce sobre los que lo cumplimentan indican que el SDS cuenta con una serie de características que estimulan su empleo y aplicación en el asesoramiento vocacional y profesional (Holland, Powell y Fritzxche, 1994; Holland y Rayman, 1986) que pasamos a citar a continuación:

1. Efectos. Holland y Raiman (1986) consideran que tan importante es la fiabilidad y la validez de los inventarios de intereses como el impacto de seleccionar un inventario de intereses, porque el inventario en sí representa una evaluación y un tratamiento vocacional. Estudios experimentales demuestran que el SDS incrementa: la autocomprensión, el número de alternativas vocacionales consideradas, la satisfacción con la elección vocacional actual y el conocimiento de la tipología de Holland. Incluso hay estudios donde se afirma que el SDS ofrece los mismos efectos beneficiosos que los orientadores profesionales.

2. Autodirección. El SDS estimula la iniciativa y el aprendizaje, ya que es el sujeto el que controla su cumplimentación. De hecho éste puede ser realizado en privado y en el momento que el sujeto lo desee. Desde este punto de vista, se puede considerar que es similar a una experiencia de aprendizaje programada. Por otro lado, el que se dé más control al cliente o usuario ayuda a que se desmitifique el proceso de orientación vocacional o profesional o de orientación para la carrera.

3. Simplicidad. Se señala como una de las características más relevantes. Todo lo necesario para obtener y usar la puntuación del SDS se encuentra en dos cuadernos 
de fácil lectura y comprensión. Por tanto, no es necesario el uso de plantillas de corrección ni de un elaborado y complejo informe de puntuación.

4. Exploración comprensiva. El SDS proporciona una completa exploración de posibilidades ocupacionales. La relación de las características personales con las 1335 ocupaciones del Descubridor de Ocupaciones es fácilmente comprensible, puesto que tanto las ocupaciones como las características personales utilizan el mismo código. Además utilizando el Dictionary of Holland Occupational Codes (DHOC) de Gottfredson y Holland (1989) se amplían a 12860 ocupaciones las que pueden ser exploradas.

5. Desarrollo personal. El SDS puede ser realizado periódicamente por la misma persona. Este hecho es consistente con una concepción del desarrollo de la orientación de la carrera. Con independencia de la edad, el lenguaje y contenido del SDS van a ser relevantes para el pensamiento vocacional.

6. Base científica. El SDS está fundamentado en una teoría de elección vocacional (Holland, 1992, 1997) que ha pasado por una extensa investigación y cuenta con una amplia aceptación.

7. Salvaguarda. Como señalan Holland, Powel y Fritzsche (1994) el SDS contiene cuatro salvaguardas para prevenir errores y resultados perjudiciales:

- El parecido de una persona con los tipos de evaluación personal viene determinado mediante cinco formas y no por una sola.

- La evaluación personal se usa de una manera conservadora. Se instruye al sujeto para buscar todas las permutaciones del código sumario de tres letras.

- El sujeto es instruido para buscar ayuda en el caso que su código sumario y su código de aspiraciones no estén en consonancia.

- Se aconseja al usuario para que consulte con un orientador cuando desee más información u otra clase de ayuda.

\section{EI uso del Self-Directed Search en el asesoramiento vocacional y profesional}

Para usar el Self-Directed Search (SDS) una persona cumplimenta el Cuaderno de Evaluación (The Assessment Booklet) y obtiene un código ocupacional o código sumario formado por tres letras. Estas tres letras representan el parecido de la persona con los seis ti- 
pos de personalidad propuestos por Holland (Realista, Investigador, Artista, Social, Emprendedor y Convencional). El código obtenido es empleado para localizar las profesiones más adecuadas al mismo, para ello se utiliza un cuaderno de clasificación de ocupaciones denominado Descubridor de Ocupaciones (The Occupations Finder). En resumen, el Self-Directed Search (SDS) proporciona una experiencia de orientación vocacional o de orientación para la carrera, sustituyendo lo que una persona y un orientador pueden hacer en el transcurso de varias entrevistas. Se puede cumplimentar de forma individual o en grupo, aunque Holland, Powel y Fritzsche (1994) consideran que es más efectivo cuando se hace en casa, en una biblioteca o en un lugar privado, de manera que se cometen menos errores, aumenta el grado de compromiso y se obtienen resultados más beneficiosos.

Cuando se cumplimenta en grupo supone una experiencia de aprendizaje colectiva a la vez que colaborativa, en la que los sujetos: aprenden rápidamente el esquema de clasificación, contribuyen con información sobre sus vidas y suelen disfrutar hablando y comentando los resultados obtenidos. Estas situaciones pueden aprovecharse para incrementar la información vocacional y sugerir nuevas líneas de exploración ocupacional, que quizás de otra manera no se hubieran tenido en cuenta. Generalmente en este tipo de situaciones los sujetos tienen una visión positiva sobre sus posibilidades vocacionales y sobre la resolución de los problemas relacionados con la elección.

Su uso puede ayudar a personas con un amplio rango de incertidumbres vocacionales entre las que se incluyen:

- Aquéllas que se preguntan qué carrera seguir.

- Aquéllas que quieren apoyar una elección previamente decidida.

- Aquéllas que quieren asegurarse de que han tenido en cuenta todas las alternativas posibles.

- Aquéllas que buscan una segunda carrera o cuestionan la adecuación de su trabajo actual.

La cumplimentación del SDS representa una experiencia de aprendizaje en la que los sujetos pueden identificar los tipos de personalidad por los que presentan una mayor afinidad y explorar un conjunto de ocupaciones o campos de estudio que más se ajustan a su perfil. Conviene que el sujeto tenga claro para qué sirve el instrumento y en qué le puede ayudar. En 
relación con su empleo en la orientación vocacional y profesional con estudiantes, sería conveniente que éste forme parte de un programa de orientación más amplio a través del cual se favorezca el desarrollo y la madurez vocacional (intereses, capacidades, motivaciones, aptitudes, autoeficacia,...), se facilite el acceso, análisis y organización de la información vocacional relacionada con el ámbito académico y profesional (estudios, itinerarios formativos, salidas profesionales, conocimiento de la profesión, mercado laboral,...) y se prepare para la toma de decisiones.

Con respecto a su empleo en el consejo y asesoramiento vocacional, la teoría de Holland (Holland, 1997; Reardon y Lent, 1998, 1999) proporciona una serie de medidas, basadas en unos supuestos secundarios, que facilitan información adicional en relación con el proceso de decisión vocacional. Estas medidas están referidas a la congruencia, la consistencia, la diferenciación, la coherencia y la frecuencia. De hecho es conveniente que el consejero u orientador fundamente su asesoramiento individualizado basándose en el análisis de estas medidas.

La congruencia viene determinada por el grado de acuerdo entre los intereses expresados en la sección de Aspiraciones Vocacionales y los intereses medidos a través de las seis escalas que evalúa el SDS. Para ello se compara el código obtenido en las Aspiraciones con el código sumario obtenido en el Cuaderno de Evaluación. Para el cálculo de la congruencia se han empleado diferentes tipos de índices (Holland, Powell y Fritzsche, 1994) como el del modelo hexagonal, el de Zener-Schnuelle, el de Iachan y el de Brown y Gore (1994). También se emplea la congruencia cuando se quiere comparar el código sumario obtenido en el SDS y un código de un determinado ambiente laboral.

La consistencia mide el grado de relación entre la configuración de la personalidad del individuo para ello se emplean las dos primeras letras obtenidas en la clave sumario. Siguiendo el modelo hexagonal de relación entre los tipos la consistencia será más elevada cuando los tipos sean adyacentes, será media cuando los tipos son alternos y baja cuando los tipos este colocados en posiciones opuestas. Por ejemplo, un tipo RIS posee una consistencia alta ya que los tipos R e I son adyacentes en el hexágono.

La diferenciación mide al grado de distinción o definición del código obtenido. Se calcula restando las puntuaciones más bajas a las más elevadas. 
La coherencia está referida a los intereses expresados en la sección de Aspiraciones. Se trata de comprobar si existe coincidencia en la primera letra del código de cada una de las ocupaciones señaladas en esta sección.

La frecuencia está determinada por el porcentaje de personas del grupo normativo que tienen el mismo código que el obtenido por un sujeto.

En general, podemos señalar que altos índices de congruencia entre aspiraciones y el código obtenido en el SDS demuestra una futura perseverancia en el tipo de elección. La consistencia alta está relacionada con más estabilidad en el historial de trabajo y en la dirección de las preferencias vocacionales. Los perfiles diferenciados son más predictibles con respecto a los intereses. Así mismo la coherencia alta también está relacionada con la persistencia en la elección. Por su parte, la frecuencia permite comparar al sujeto con una muestra normativa que tiene en cuenta la edad, el sexo y la educación.

Todos estos supuestos se pueden tener en cuenta en el asesoramiento sobre todo cuando nos podemos encontrar en un extremo, sujetos que manifiesten una congruencia baja, una consistencia baja, una escasa diferenciación del perfil y una coherencia baja. Esto llevaría al asesor a realizar un análisis más exhaustivo del caso y a una asistencia técnica individualizada. Claro está que se pueden dar diferentes tipos de casos y, en consecuencia, deberan recibir un asesoramiento acorde con los resultados obtenidos en los supuestos secundarios.

Por otra parte, las distintas secciones (Actividades, Habilidades, Ocupaciones, Autoevaluaciones) que aparecen en el SDS también pueden ofrecen información adicional que puede usarse en el asesoramiento en tanto en cuanto, cada una de ellas ofrecen puntuaciones en cada uno de los seis tipos; lo que permite comprobar el grado de congruencia que se dan entre ellas.

Otro aspecto que se está considerando como indicador a tener en cuenta en el asesoramiento y la toma de decisiones sobre la carrera es el constructo referido a la elevación del perfil. Bullock y Reardon (2005) lo definen como la suma de las seis puntuaciones de los tipos RIASEC correspondientes a las cinco secciones del SDS (Actividades, Habilidades, Ocupaciones, Autoevaluaciones 1, Autoevaluaciones 2). Como señalan estos autores esta puntua- 
ción puede proporcionar información adicional con respecto a la personalidad, las preferencias, las tendencias y las necesidades de los sujetos asesorados. Un perfil alto está relacionado moderadamente con variables positivas de la personalidad y un buen funcionamiento educativo. También es predictor de un estilo expresivo, entusiasta o impulsivo. Es probable que las personas que obtengan puntuaciones altas tengan una disposición agradable y optimista, siendo expresivos en sus pensamientos, necesidades y preferencias. En este sentido Gottfredson y Jones (1993) mencionan que estos sujetos suelen tener un estilo de decisión impulsivo que el consejero o asesor debe tener en cuenta.

Por otro lado un perfil bajo está relacionado con rasgos depresivos; se presentan como personas reservadas, poco entusiasmadas y expresivas. Sujetos con este perfil puede que tenga deseo de expresar sus necesidades, pensamientos, y preferencias, aspectos que tienen implicaciones importantes para los consejeros y orientadores de la carrera.

Para finalizar, señalar que el empleo del SDS en el asesoramiento va a permitir a los orientadores:

- Interpretar puntuaciones de intereses de acuerdo con unas formulaciones teóricas.

- Realizar inferencias a partir de las medidas obtenidas de los supuestos secundarios que van a facilitar una información complementaria y necesaria en el asesoramiento.

- Predecir las categorías de ocupaciones que parecen congruentes con una persona.

- Obtener información sobre las características de personalidad de los asesorandos.

- Identificar fuentes de trabajo satisfactorio o insatisfactorio, empleando las fórmulas de congruencia entre persona y trabajo.

- Identificar sujetos cuyas características individuales indican una necesidad de orientación vocacional y profesional.

- Iniciar un proceso exploración y de toma de decisiones sobre la carrera. 


\section{Conclusiones}

A modo de síntesis señalar que el SDS representa una guía para la planificación de la carrera que se fundamenta en una teoría (Holland, 1997) cuya dimensión aplicada es ampliamente reconocida. La información que nos ofrece va más allá que la de un inventario de intereses. En ese sentido proporciona información acerca de rasgos de personalidad, preferencias vocacionales y profesionales, metas de vida, valores, competencias, historia personal y autoeficacia. Por otra parte, el hecho de ser un instrumento autoaplicable, autopuntuable y autointerpretable lo ditinguen de otras herramientas de orientación vocacional y profesional.

Aunque como señalan Holland, Powel y Fritzsche (1994) fue desarrollado para tratar problemas vocacionales se le han encontrado otras aplicaciones relacionadas con la asistencia vocacional, la organización de datos ocupacionales y personales y la investigación.

En general su uso, tanto a nivel individual como grupal, representa una experiencia de aprendizaje a través de la cual el sujeto se inicia en un proceso de exploración y de toma de decisiones relacionadas con el desarrollo de la carrera. 


\section{Referencias bibliográficas}

Balkis, M. y Isiker, G. ( 2005). The relationship between thinking styles and personality types. Social Behavior and Personality, 33(3), 283-294.

Bath, A. (2004). The relationship between person-environment congruence and fundamental goals for African-American and European-American, female college students. Dissertation Abstracts International, 64(10-B), 5264.

Barak, A. y Cohen, L. (2002). Empirical examination of an online version of the SelfDirected Search. Journal of Career Assessment, 10(4), 387-400.

Brown, S. D., y Gore, P. A., Jr. (1994). An evaluation of interest congruence indices: Distribution characteristics and measurement properties. Journal of Vocational Behavior, $45,310-327$.

Bullock, E. y Reardon, R. (2005). Using Profile Elevation to Increase the Usefulness of the Self-Directed Search and Other Inventories. The Career Development Quarterly, 54 (2), 175-184.

Campbell, D. P. y Holland J. (1972). A merger in vocational interest research: Applying Holland's theory to Strong's data. Journal of Vocational Behavior, 2, 353-376.

Dockins, J. (2004). Person-environment congruence, job stability, and job satisfaction: An examination of Holland's theory of vocational personalities and work environments in the nursing profession (John L. Holland). Dissertation Abstracts International, 65 (3A), 795 .

Einarsdottir, S., Rounds, J., Aegisdottir, S. y Gerstein, L. (2002). The structure of vocational interests in Iceland: Examining Holland's and Gati's RIASEC models. European Journal of Psychological Assessment, 18(1), 85-95.

Glaser, B., Calhoun, G., Bates, J. y Bradshaw, C. (2003). Self-reported career interests among adjudicated male adolescents: A pilot study. Journal of Addictions and Offender Counseling. 23(2), 73-82.

Gottfredson, G. y Holland, J. L. (1989). Dictionary of Holland Occupational Codes. Odessa, FL: Psychological Assessment Resources.

Gottfredson, G. y Holland, J. L. (1991). Position Classification Inventory. Item booklet. Odessa, FL: Psychological Assessment Resources.

Gottfredson, G. D., y Jones, E. M. (1993). Psychological meaning of profile elevation in the Vocational Preference Inventory. Journal of Career Assessment, 1, 35-49. 
Hoffman, E. (2005). Relationships between personality characteristics and nontraditional career choices of women in Northwestern Minnesota based on Holland's Hexagon Model (John 1. Holland). Dissertation Abstracts International, 66(6-A), 2113.

Holland, J. L. (1958). A personality inventory employing occupational titles. Journal of Applied Psychology, 42, 336-342.

Holland, J. L. (1959). A theory of vocational choice. Journal of Counseling Psychology, 6, $35-45$.

Holland, J. L. (1963). Explorations of a theory of vocational choice and achievement: II. A four year prediction study. Psychological Reports, 12, 537-594.

Holland, J. L. (1966). A psychological classification scheme for vocations and majors fields. Journal of Counseling Psychology, 13, 278-288.

Holland, J. L. (1971). A theory-riddencomputerless, impersonal vocational guidance system. Journal of Vocational Behavior, 1, 167-176.

Holland, J. L. (1975). La elección vocacional. Teoría de las carreras. México: Trillas.

Holland, J. L. (1981). Técnica de la elección vocacional. Tipos de personalidad y modelos ambientales. México: Trillas.

Holland, J. L. (1992). Making vocational choices: A theory of vocational personalities and work environments, 2er ed. Odessa, FL: Psychological Assessment Resources.

Holland, J. L. (1995a). Cuaderno de Evaluación. Una guía para la planificación educacional y vocacional. Odessa FL: Psychological Assessment Resources.

Holland, J. L. (1995b). Descubridor de Ocupaciones. Odessa FL: Psychological Assessment Resources.

Holland, J. L. (1995c). Usted y su Carrera. Odessa FL: Psychological Assessment Resources.

Holland, J. L. (1997). Making vocational choices: A theory of vocational personalities and work environments, 3er ed. Odessa, FL: Psychological Assessment Resources.

Holland, J. L., Fritzsche, B. y Powel, A. (1994). Sel-Directed Search. Technical manual. Odessa, FL: Psychological Assessment Resources.

Holland, J. L. y Raiman, J. (1986). The Self-Directed Search. En Walsh, B; Osipow, S. Advances in Vocational Psychology. London: Lawrence Erlabaum Associates.

Holland, J. L., Powel, A.y Fritzsche, B. (1994). Self-Directed Search. Professional users's guide. Odessa, FL: Psychological Assessment Resources.

Holland, J. L., Whitney, D. R., Cole, N. S., Richards, J. M. (1969). An empirical occupational classification derived from a theory of personality and intended for practice and re- 
search (ACT Research Report $\left.n^{\circ} 29\right)$. Iowa City: The American College Testing Program.

Jones, L., Sheffield, D. y Joyner, B. (2000). Comparing the effects of the Career Key with Self-Directed Search and Job-OE among eigth-grades students. Professional School Counseling, 3(4), 238-247.

Labarbera, D. (2005). Physician Assistant Self-Directed Search Holland Codes. Journal of Career Assessment, 13(3), 337-346.

Leung, S. A. y Hou, Z. J. (2001). Concurrent validity of the 1994 Self-Directed Search for Chinese high school students in Hong Kong. Journal of Career Development, 9(3), 283-296.

Leung, S. A. y Hou, Z. J. (2005). The Structure of Vocational Interests Among Chinese Students. Journal of Career Development, 32(1), 74-90.

Lowman, R., Palmer, L., Santana, R. y Abott, J. (2003). Executive and Career Assessment: Can Alternative Occupational Interest Measures Be Used Interchangeably?. Psychologist Manager Journal, 6(2), 65-78.

Lumsden, J., Sampson, J., Reardon, R., Lenz, J. y Peterson, G. (2004). A Comparison Study of the Paper-and-Pencil, Personal Computer, and Internet Versions of Holland's SelfDirected Search. Measurement and Evaluation in Counseling and Development, 37(2), $85-94$

Mahalik, J. (2001). An exploration of the relationship between Holland's personality typology and personality disorder traits: A self-concept perspective (John L. Holland). Dissertation Abstracts International, 62(6-B), 2985.

Martínez-Vicente, J. M. (2001). Estudio y Adaptación del Self-Directed Search Form R (SDS) $y$ del Position Classification Inventory (PCI) a la población española. Almería: Servicio de publicaciones de la Universidad de Almería.

Martínez-Vicente, J. M. y Valls, F. (2006). La elección vocacional y la planificacion de la carrera. Adaptacion española del Self-Directed Search (SDS-R) de Holland, Psicothema, 18 (1), 117-122.

Martínez-Vicente, J. M., Valls, F. y Álvarez, J. (2003). La personalidad en la conducta vocacional. En F. Rivas (Ed.), Asesoramiento vocacional. Teoría, práctica e instrumentación. Barcelona: Ariel.

McCormick, E. J., Jeanneret, P. R. y Mechan, R. C. (1969). The used of data based on the Position Analysis Questionnaire in developing synthetically-derivied attribute re- 
quirements of jobs. Office of Naval Research Contract Nonr-1100 (28), Informe $\mathrm{n}^{0} 4$. Lafayette, Ind: Occupational Research Center, Purdue University.

Miller, M., Springer, T., Tobacyk, J. y Wells, D. (2004). Congruency between occupational daydreams and SDS scores among college students. College Student Journal. 38 (1). 57-60.

Muck, P. (2005). EXPLORIX. Deutschsprachige Adaptation und Weiterentwicklung des Self-directed Search nach Holland. Zeitschrift fur Personalpsychologie, 4(1) 2005, 3946.

Osborn, D. y Reardon, R. (2006). Using the Self-Directed Search: Career Explorer With High-Risk Middle School Students. Career Development Quarterly, 54(3), 269-273.

Price, P. (2003). Values of engineering majors: A step beyond Holland's model (John L. Holland). Dissertation Abstracts International, 63(7-A), 2459.

Primi, R., Pellegrini, M., Porto. D., Hernandez-Munhoz, A., Bighetti, C. y Moggi, M. (2001) Caracteristicas del personalidade los e indecisao profissional / Personalidad rasgos y indecisión de la carrera. Psico, 32(1), 81-96.

Reardon, R. y Lenz, J. (1998). The Self-Directed Search and related Holland career materials: A practitioner's guide. Odessa, FL: Psychological Assessment Resources.

Reardon, R. y Lenz, J.(1999). Holland's theory and career assessment. Journal Vocational Behavior, 50, 102-113.

Strong, E. K. (1943). Vocational Interests of Men and Woman. Stanford, CA: Stanford University Press.

Savickas, M., Taber, B. y Spokane, A. (2002). Convergent and discriminant validity of five interest inventories. Journal of Vocational Behavior, 61(1), 139-184.

Sverko, I. y Babarovic, T (2006). The Validity of Holland's Theory in Croatia. Journal of Career Assessment, 14(4), 490-507.

Swan, K. Classifying personality types and determining a profile of female carpenters based on Holland's hexagon model.Dissertation Abstracts International, 63(5-B), 2636.

Wilkes, L. (2002). A study of job performance of young adults with disabilities comparing those matched and not matched using the Holland self-directed search. Dissertation Abstracts International. 63(2-B), 1078.

Yahney, L. (2005). Differences between personality typology and motivational cuing among exercise groups. Dissertation Abstracts International, 65(12-A), 4478.

Yang, W., Stokes, G. y Huy, C. (2005). Cross-cultural validation of Holland's interest structure in Chinese population. Journal of Vocational-Behavior, 67(3), 379-396. 
Zhang, L. (2000). Are thinking styles and personality types related? Educational Psychology, 20(3), 271-282.

Zhang, L. (2001). Thinking styles and personality types revisited. Personality and Individual Differences, 31(6), 883-894.

Zhang, L. (2004). Learning approaches and career personality types: Biggs and Holland united. Personality and Individual Differences, 37(1), 65-81. 